\title{
Aarco Fatores intervenientes nos indicativos de depressão em idosos usuários das unidades básicas de saúde de Maringá, Paraná, 2017* \\ doi: $10.5123 /$ S1679-49742019000300010
}

\author{
Factors influencing depression markers in elderly primary healthcare center patients in \\ Maringá, Paraná, Brazil, 2017
}

\section{Factores intervienientes en los indicativos de depresión en adultos mayores usuarios de las unidades básicas de salud de Maringá, Paraná, Brasil, 2017}

\author{
Daniel Vicentini de Oliveira ${ }^{1}$ - (1) orcid.org/0000-0002-0272-9773 \\ Naelly Renata Saraiva Pivetta ${ }^{1}$ - (10 orcid.org/0000-0002-2822-4618 \\ Gustavo Vinicius do Nascimento de Oliveira² - orcid.org/0000-0003-3017-467X \\ Diogo Alves da Silva² - (D) orcid.org/0000-0002-9530-2073 \\ José Roberto Andrade do Nascimento Júnior ${ }^{3}$ - (1) orcid.org/0000-0003-3836-6967

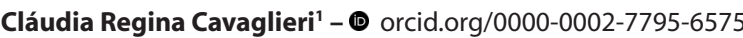 \\ ${ }^{1}$ Universidade Estadual de Campinas, Departamento de Pós-Graduação Stricto Sensu em Gerontologia, Campinas, SP, Brasil \\ ${ }^{2}$ Centro Universitário Metropolitano de Maringá, Departamento de Graduação em Educação Física, Maringá, PR, Brasil \\ ${ }^{3}$ Universidade Federal do Vale do São Francisco, Departamento de Pós-Graduação Stricto Sensu em Educação Física, Petrolina, \\ PE, Brasil
}

\begin{abstract}
Resumo
Objetivo: analisar os fatores intervenientes nos indicativos de depressão em idosos das unidades básicas de saúde (UBS) do município de Maringá, Paraná, Brasil, em 2017. Métodos: estudo transversal, realizado com idosos usuários das UBS de Maringá; utilizou-se um questionário com questões sociodemográficas, a Escala de Depressão Geriátrica (Geriatric Depression Scale [GDS]) e o Questionário Internacional de Atividade Física (International Physical Activity Questionnaire [IPAQ]); foram analisadas as associações e comparações do indicativo de depressão com as variáveis sociodemográficas e as condições de saúde. Resultados: 654 idosos participaram do estudo; apresentaram maior indicativo de depressão aqueles com menor renda mensal, percepção de saúde ruim, histórico de quedas e três ou mais comorbidades, enquanto os fisicamente ativos apresentaram menor indicativo de depressão. Conclusão: renda mensal e condições de saúde parecem ser fatores intervenientes nos indicativos de depressão; a prática de atividades físicas leves está associada a menor tendência de depressão na terceira idade.
\end{abstract}

Palavras-chave: Atividade Motora; Depressão; Estudos Transversais; Geriatria; Envelhecimento.

*Estudo financiado com recursos da Coordenação de Aperfeiçoamento de Pessoal de Nível Superior, Ministério da Educação (Capes/MEC): Processo n 01P-3372/2017.

Endereço para correspondência:

Daniel Vicentini de Oliveira - Universidade Estadual de Campinas, Rua Tessália Vieira de Camargo, nº 126, Cidade Universitária

Zeferino Vaz, Campinas, SP, Brasil. CEP: 13083-887 Caixa Postal 464

E-mail: d.vicentini@hotmail.com 


\section{Introdução}

0 envelhecimento é um processo dinâmico e progressivo, caracterizado por alterações funcionais, bioquímicas, fisiológicas, morfológicas e psicológicas. ${ }^{1}$ Essas alterações levam o organismo do indivíduo a ter reduzida, gradativamente, sua capacidade de resposta e recuperação, frente a agentes estressores, e de adaptação ao meio, ${ }^{2}$ de tal forma que $o$ indivíduo se torna mais vulnerável e suscetível a doenças, lesões, incapacidades e morte. ${ }^{2}$

\section{A UBS éo espaço oportuno e ideal para triagem da depressão e conhecimento do perfil socioeconômico e de saúde de idosos da comunidade por ela atendida.}

0 aumento no número de anos vividos é, sem dúvida, uma conquista, sobretudo em países de renda média e baixa. ${ }^{3}$ No entanto, viver mais não é sinônimo de viver melhor. ${ }^{2} 0$ envelhecimento pode estar associado ao declínio funcional e cognitivo, perda da autonomia e independência, privação do papel social, improdutividade, isolamento, carência afetiva, frustrações, sentimento de culpa, de inutilidade e depressão. ${ }^{2}$ Isto pode ocorrer porque essa é a fase da vida quando, literalmente, o idoso reflete sobre as escolhas do passado e pondera os anos vividos, e, ao mesmo tempo, busca maneiras de lidar com o envelhecimento e as condições adversas que possa enfrentar em decorrência do avanço da idade. Neste contexto, sintomas depressivos podem surgir. ${ }^{1}$

A depressão tornou-se comum entre a população idosa. ${ }^{4}$ Em todo o mundo, cerca de $15 \%$ dos idosos apresentam pelo menos um sintoma depressivo. ${ }^{4}$ Segundo a Organização Mundial da Saúde (OMS), a depressão é um problema de Saúde Pública. ${ }^{4}$ Ela se caracteriza como um transtorno de humor, seguido de redução progressiva do interesse por atividades sociais, irritação, apatia, diminuição da sensação de prazer, pessimismo e alterações do sono e do apetite. ${ }^{4}$

Estudos apontam que doenças crônicas, sexo feminino e perda da independência funcional são fatores que contribuem para o aumento de sintomatologia depressiva na terceira idade. ${ }^{4}$ Igualmente, há evidências de que menores níveis educacionais e de renda mensal estão associados a maior ocorrência de depressão, em consonância com outros estudos conduzidos no Brasil. ${ }^{5}$ Todos esses fatores são determinantes sociais de saúde: características socioeconômicas e demográficas e a ausência de rede social de apoio aumentam o risco da ocorrência da depressão. ${ }^{5}$

A depressão em idosos representa um desafio ao Sistema Único de Saúde (SUS), cujos profissionais devem estar atentos ao rastreio desse distúrbio. Ao SUS também cabe oferecer apoio social, farmacológico e psicológico aos idosos. ${ }^{5}$

No âmbito do sistema público, a unidade básica de saúde (UBS) é o local onde acontece o primeiro contato do usuário com o atendimento em saúde. 0 objetivo da UBS é atender a $80 \%$ dos problemas de saúde da população, evitando o encaminhamento para outros serviços, como emergências e hospitais, promover a saúde e prevenir doenças. ${ }^{6}$ Portanto, a UBS é o espaço oportuno e ideal para triagem da depressão e conhecimento do perfil socioeconômico e de saúde de idosos da comunidade por ela atendida. Nos países de renda média e baixa, estudos sobre a depressão em idosos e seus fatores associados ainda são escassos, justificando a necessidade de mais pesquisas exploradoras dessa temática. ${ }^{7}$

0 presente estudo teve como objetivo analisar os fatores intervenientes nos indicativos de depressão em idosos das unidades básicas de saúde do município de Maringá, Paraná, Brasil, no ano de 2017.

\section{Métodos}

Estudo epidemiológico, de corte transversal e base populacional, realizado entre maio e setembro de 2017.

Segundo dados obtidos junto à Secretária Municipal de Saúde de Maringá, em 2016, a população-alvo do estudo era composta por 42.258 idosos. A amostra inicial do estudo foi estimada considerando-se um nível de confiança de $95 \%$ e 4 pontos percentuais de margem de erro, e uma prevalência estimada de $50 \%$, correspondendo a 595 idosos. Adicionando-se 10\% de possíveis perdas, a amostra final foi de 654 idosos de ambos os sexos. 0 software utilizado para o cálculo de tamanho da amostra foi o StatDisk versão 8.4 .

As UBS nas quais são atendidos os idosos questionados foram subdivididas em quatro regiões: Leste (7 UBS), que concentra $21,8 \%$ da população idosa; Norte (8 UBS), com 34,6\%; Oeste (8 UBS), com 23,2\%; e Sul 
(8 UBS), contemplando $20,4 \%$ da população total de idosos da cidade. Em cada uma dessas regiões, foram selecionadas - por sorteio - três UBS para serem avaliadas (Figura 1).

Após o sorteio das UBS a serem investigadas, a proporção de idosos habilitados a participar foi proporcional a cada uma das UBS selecionadas; em outras palavras, o tamanho do contingente investigado foi proporcional ao número de idosos nas UBS, haja vista ser importante manter a proporção de idosos da população na amostra. Pela mesma razão, os cálculos para obtenção da amostra final por UBS de acordo com o sexo também foram proporcionais aos cálculos populacionais.

Foram incluídos no estudo idosos de ambos os sexos, com idade igual ou superior a 60 anos, capacidade de fala e audição preservadas, de maneira a permitir a aplicação dos questionários. Foi utilizado o miniexame do estado mental (MEEM), para excluir idosos com deficits cognitivos importantes. O MEEM é composto por questões agrupadas em sete categorias: (i) orientação temporal (escore máximo: 5); (ii) orientação espacial (escore máximo: 5); (iii) registro de três palavras (esco- re máximo: 3); (iv) atenção e cálculo (escore máximo: 5); (v) recordação das três palavras - evocação (escore máximo: 3); (vi) linguagem (escore máximo: 8); e (vii) capacidade visuoconstrutiva (escore máximo: 1 )

Quanto maior o escore em cada domínio, melhor o estado cognitivo no mesmo. ${ }^{8}$ As notas de corte utilizadas para exclusão pelo MEEM foram: 17 para os analfabetos; 22 para idosos com escolaridade entre 1 e 4 anos de estudo; 24 para aqueles com escolaridade entre 5 e 8 anos; $\mathrm{e} 26$ para os que tiverem 9 ou mais anos de estudo. Estes pontos de corte foram baseados nos critérios de Brucki et al. ${ }^{9} \mathrm{e}$ correspondem à média obtida por esses autores para cada faixa de escolaridade, menos um desvio-padrão. Idosos classificados abaixo do ponto de corte específico para sua escolaridade foram excluídos.

Para a caracterização do perfil sociodemográfico e de saúde dos idosos, utilizou-se um questionário semiestruturado, destinado à coleta das seguintes informações:

a) idade (em anos: 60 a 69; 70 a 79; 80 ou mais);

b) sexo (masculino; feminino);

c) estado civil (casado [ou vivendo com companheiro(a)]; solteiro; divorciado/separado/viúvo);

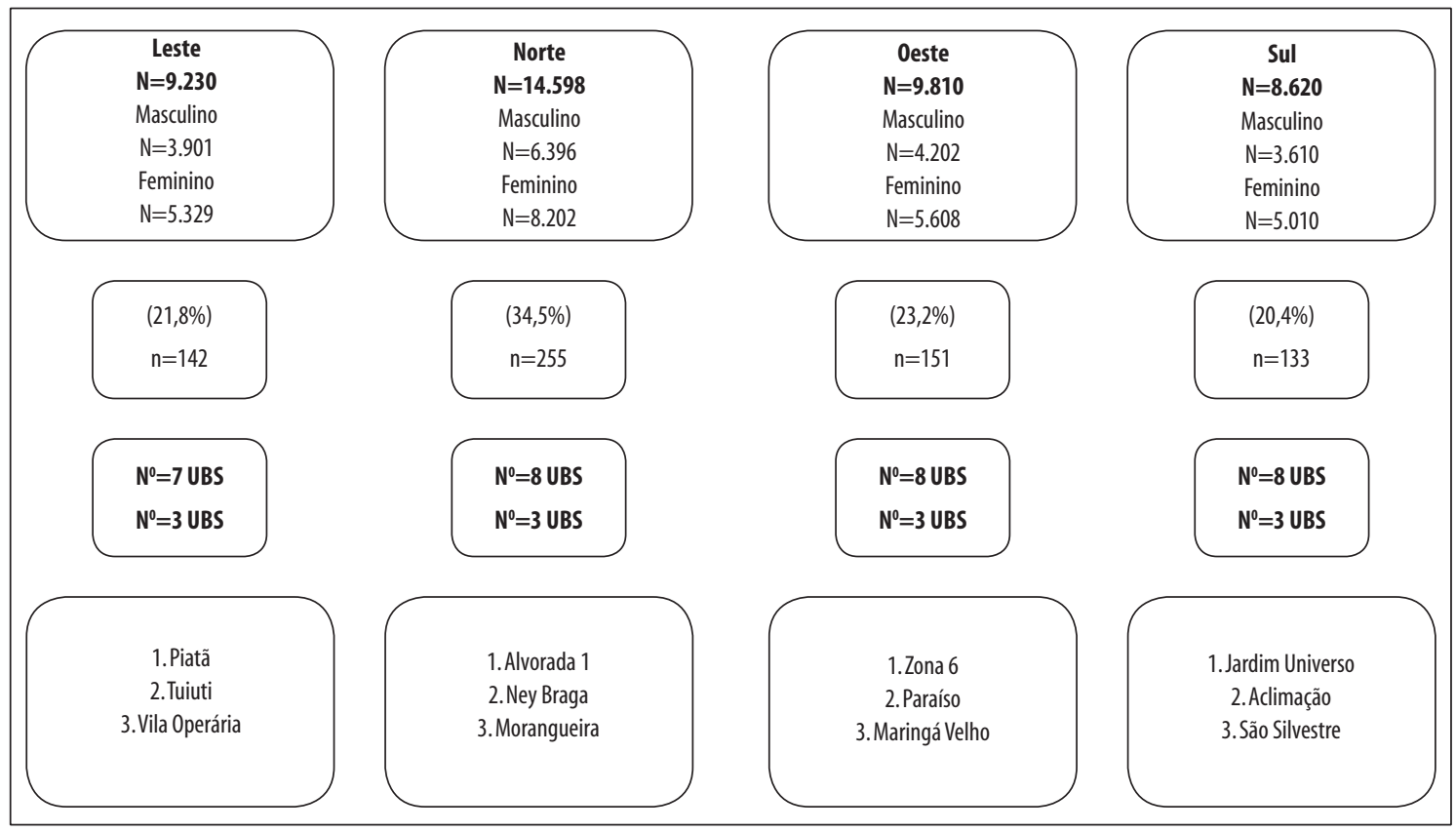

UBS: unidade básica de saúde.

Figura 1 - Diagrama da amostragem realizada com os idosos usuários das unidades básicas de saúde, Maringá, Paraná, 2017 
d) raça/cor da pele (branca; preta; outra);

e) renda mensal (em salário mínimo [SM] de referência no ano de 2016, R\$ 880,00: 1 a 2 SM; 2,1 a 3 SM; mais de $3 \mathrm{SM}$ );

f) aposentadoria (sim; não);

g) escolaridade (não estudou; ensino fundamental incompleto; ensino fundamental completo; ensino médio completo; ensino superior);

h) autopercepção do estado de saúde (ruim; regular; bom; muito bom);

i) quantidade de medicamentos utilizados (nenhum; 1 a 2; mais de 2);

j) histórico de queda ou quase queda no último semestre (sim; não); $\mathrm{e}$

k) comorbidades presentes (nenhuma; 1 a $2 ; 3$ ou mais)

0 nível de atividade física dos idosos foi avaliado utilizando-se a versão curta do Questionário Internacional de Atividade Física (International Physical Activity Questionnaire [IPAQ]). Este questionário é composto por sete questões abertas e suas informações permitem estimar o tempo despendido por semana/por dia/por minuto em diferentes dimensões de atividade física (caminhadas e esforços físicos de intensidades moderada e vigorosa) e de inatividade física (posição sentada). 0 nível de atividade física foi classificado em quatro estratos:

(i) Sedentário - idoso que não realizou nenhuma atividade física por pelo menos 10 minutos contínuos durante a semana.

(ii) Irregularmente ativo - idoso que realizou atividade física, porém de forma insuficiente para ser classificado como ativo porque não cumpria as recomendações quanto à frequência ou duração dessa atividade.

(iii) Ativo - idoso que realizava pelo menos 3 dias de atividade vigorosa, por no mínimo 20 minutos; ou 5 dias ou mais de atividade moderada ou caminhada por no mínimo 30 minutos; ou pelo menos 5 dias e 150 minutos semanais de qualquer tipo de atividade física.

(iv) Muito ativo - idoso que realizava atividades vigorosas por pelo menos 5 dias na semana, sendo 30 minutos por sessão; ou no mínimo 3 dias na semana de atividade vigorosa, no mínimo 20 minutos por sessão, mais atividades moderadas ou caminhada, por no mínimo 5 dias na semana e 30 minutos por sessão.
O comportamento sedentário foi avaliado pelo tempo médio sentado em um dia de semana, e em um dia de final de semana. ${ }^{10}$

0 instrumento da Escala de Depressão Geriátrica (Geriatric Depression Scale [GDS]), em sua versão curta, foi utilizado para detecção dos escores indicativos de depressão. A GDS apresenta 15 perguntas negativas/ afirmativas, cujo resultado de cinco ou mais pontos é indicativo de depressão. ${ }^{11}$

A coleta foi realizada por três pesquisadores treinados, um mês antes, por um fisioterapeuta, pesquisador responsável pelo projeto. 0 treinamento incluiu um estudo piloto com 30 idosos que não participaram do estudo propriamente dito. Foram abordados os idosos voluntários que se encontravam na sala de espera da UBS. Para aplicação dos questionários, optou-se pela entrevista direta em razão da possível dificuldade de leitura, problemas visuais e de compreensão das questões propostas. A UBS não alterou seu modelo tradicional de atendimento, durante a abordagem da pesquisa dentro da própria unidade; a única condição apresentada foi de que os idosos não passassem por esse atendimento sem antes serem entrevistados. A duração média das entrevistas foi de 15 minutos.

Inicialmente, foi verificada a normalidade dos dados por meio do teste Kolmogorov-Smirnov. Como os dados não apresentaram distribuição normal, foram utilizadas a mediana (Md) e o intervalo interquartil como medidas descritivas. Na comparação do indicativo de depressão em função das variáveis sociodemográficas e das condições de saúde, foram utilizados os testes de Kruskal-Wallis e ' $U$ ' de Mann-Whitney. A correlação entre o nível de atividade física e 0 indicativo de depressão foi efetuada por meio do coeficiente de Spearman. Considerou-se um nível de significância de $\mathrm{p}<0,05$. Todas as análises foram realizadas com uso do software Statistical Package for the Social Sciences (SPSS) versão 22.0.

0 projeto do estudo foi aprovado pelo Comitê de Ética em Pesquisa do Centro Universitário de Maringá (CEP/ UniCesumar): Parecer n $n^{0}$ 1.626.966/2016. Na UBS, os idosos foram abordados pelo pesquisador responsável ou pela equipe de pesquisadores, informados sobre a justificativa, objetivos e procedimentos do estudo, conforme as orientações para pesquisa com seres humanos constantes na Resolução do Conselho Nacional de Saúde (CNS) $\mathrm{n}^{0}$ 466, de 12 de dezembro de 2012. Aqueles que aceitaram participar da pesquisa assinaram o Termo de Consentimento Livre e Esclarecido (TCLE). 


\section{Resultados}

Dos 654 idosos entrevistados, a maioria era do sexo feminino $(56,0 \%)$, casados $(61,3 \%)$, com idade entre 60 e 69 anos (59,2\%), renda mensal de 1 a 2 salários mínimos $(70,0 \%)$, nunca fumaram $(56,6 \%)$, de raça/ cor da pele branca $(81,0 \%)$ e aposentados $(75,0 \%)$. Quanto à escolaridade, também se observou que a parcela mais significativa era de idosos com ensino fundamental incompleto (43,0\%).

Em relação ao perfil de saúde, $48,5 \%$ possuíam percepção de sua saúde como boa, $44,8 \%$ procuraram a UBS para visita médica, 43,9\% tomavam de 1 a 2 medicamentos regularmente, $83,7 \%$ não tiveram histórico de quedas e $79,7 \%$ referiram quase-quedas nos seis meses anteriores à entrevista. Uma proporção maior de idosos (60,7\%) apresentou nível de atividade física ativo/muito ativo. A maioria relatou 1 a 2 morbidades associadas (50,5\%) e não apresentou indicativos de depressão $(70,6 \%)$. De uma forma geral, os idosos apresentaram mediana de 3,0 (Q1=1,0;Q3=6,0) no escore de depressão, revelando baixo indicativo de depressão (Tabela 1).

Verificou-se diferença estatisticamente significativa apenas para a renda mensal $(\mathrm{p}=0,001)$, indicando que os idosos com renda mensal acima de três salários mínimos possuíam menor indicativo de depressão do que os idosos com menor poder aquisitivo (Tabela 1).

Ao comparar o indicativo de depressão dos idosos em função das condições de saúde (Tabela 2), verificou-se diferença significativa para a autopercepção do estado de saúde $(\mathrm{p}=0,001)$, uso de medicamentos

\section{Tabela 1 - Comparação do indicativo de depressão dos idosos usuários das unidades básicas de saúde em função das variáveis sociodemográficas, Maringá, Paraná, 2017}

\begin{tabular}{|c|c|c|}
\hline \multirow{2}{*}{ Variáveis } & Indicativo de depressão & \multirow{2}{*}{$\mathbf{p}$} \\
\hline & $\operatorname{Md}(Q 1 ; Q 3)$ & \\
\hline \multicolumn{3}{|l|}{$\operatorname{Sexo}^{\mathrm{a}}$} \\
\hline Masculino & $3,0(1,0 ; 6,0)$ & \multirow[t]{2}{*}{0,134} \\
\hline Feminino & $3,0(1,0 ; 6,0)$ & \\
\hline \multicolumn{3}{|l|}{ Faixa etária (em anos) ${ }^{b}$} \\
\hline $60-69$ & $2,0(1,0 ; 6,0)$ & \multirow{3}{*}{0,085} \\
\hline $70-79$ & $3,0(1,0 ; 6,0)$ & \\
\hline$\geq 80$ & $3,0(0,0 ; 5,0)$ & \\
\hline \multicolumn{3}{|l|}{ Estado civil $^{b}$} \\
\hline Casado & $3,0(1,0 ; 6,0)$ & \multirow[t]{3}{*}{0,249} \\
\hline Solteiro & $2,0(1,0 ; 7,0)$ & \\
\hline Divorciado/separado/viúvo & $3,0(2,0 ; 7,0)$ & \\
\hline \multicolumn{3}{|c|}{ Renda mensal (em salários mínimos [SM]) } \\
\hline 1 a $2 S M$ & $3,0(1,0 ; 6,0)$ & \multirow{3}{*}{$0,001^{c}$} \\
\hline 2,1 a $3 \mathrm{SM}$ & $3,0(1,0 ; 6,0)$ & \\
\hline Mais de 3 SM & $1,0(0,0 ; 3,0)$ & \\
\hline \multicolumn{3}{|l|}{ Aposentadoria $^{\mathrm{a}}$} \\
\hline Sim & $3,0(1,0 ; 6,0)$ & \multirow[t]{2}{*}{0,255} \\
\hline Não & $3,0(1,0 ; 7,0)$ & \\
\hline
\end{tabular}

a) Teste ' $U$ ' de Mann-Whitney.

b) Teste de Kruskal-Wallis: Faixa etária - Mais de 3 SM, com 1 a 2 SM e 2,1 a 3 SM

c) Diferença significativa: $p<0,05$.

$M d=$ mediana.

$S M=$ salário mínimo 
$(\mathrm{p}=0,001)$, histórico de quedas $(\mathrm{p}=0,003)$, nível de atividade física $(\mathrm{p}=0,027)$ e comorbidades associadas $(\mathrm{p}=0,001)$. Tais achados evidenciaram que os idosos com autopercepção de saúde ruim e que tomavam mais de 2 medicamentos regularmente apresentaram maior indicativo de depressão que os idosos com autopercepção de saúde regular e boa e que tomavam menos medicamentos (nenhum; 1 a $2)$, respectivamente.
Destaca-se também (Tabela 2) que os idosos com histórico de quedas e com 3 ou mais comorbidades associadas possuíam maior indicativo de depressão quando comparados aos idosos sem histórico de quedas e com menos comorbidades associadas (nenhuma; 1 a 2), respectivamente. Em relação ao nível de atividade física, os idosos muito ativos/ ativos fisicamente apresentaram menor indicativo de depressão do que os irregularmente ativos.

\section{Tabela 2 - Comparação do indicativo de depressão dos idosos usuários das unidades básicas de saúde em função das condições de saúde, Maringá, Paraná, 2017}

\begin{tabular}{|c|c|c|}
\hline \multirow{2}{*}{ Variáveis } & Indicativo de depressão & \multirow{2}{*}{$\mathbf{p}$} \\
\hline & $\operatorname{Md}(Q 1 ; Q 3)$ & \\
\hline \multicolumn{3}{|c|}{ Autopercepção do estado de saúde } \\
\hline Boa & $2,0(1,0 ; 4,0)$ & \multirow{3}{*}{$0,001^{d}$} \\
\hline Regular & $3,0(1,0 ; 6,0)$ & \\
\hline Ruim & $6,0(2,0 ; 9,0)$ & \\
\hline \multicolumn{3}{|l|}{ Motivo da ida à UBS ${ }^{a}$} \\
\hline Visita médica & $2,0(1,0 ; 6,0)$ & \multirow{3}{*}{0,158} \\
\hline Buscar medicamentos & $3,0(1,0 ; 7,0)$ & \\
\hline Realização de exames & $3,0(1,0 ; 5,0)$ & \\
\hline \multicolumn{3}{|l|}{ Uso de medicamentos ${ }^{\mathrm{a}}$} \\
\hline Nenhum & $2,0(1,0 ; 3,8)$ & \multirow{3}{*}{$0,001^{d}$} \\
\hline $1 \mathrm{a} 2$ & $2,0(1,0 ; 5,0)$ & \\
\hline Mais de 2 & $4,0(2,0 ; 7,0)$ & \\
\hline \multicolumn{3}{|l|}{ Histórico de quedas ${ }^{\mathrm{b}}$} \\
\hline $\operatorname{Sim}$ & $4,0(2,0 ; 7,0)$ & \multirow{2}{*}{$0,003^{d}$} \\
\hline Não & $3,0(1,0 ; 6,0)$ & \\
\hline \multicolumn{3}{|c|}{ Histórico de quase-quedas ${ }^{\mathrm{b}}$} \\
\hline $\operatorname{Sim}$ & $3,0(1,0 ; 6,0)$ & \multirow{2}{*}{0,322} \\
\hline Não & $3,0(1,0 ; 6,0)$ & \\
\hline \multicolumn{3}{|l|}{ Nível de atividade física ${ }^{\mathrm{a}}$} \\
\hline Muito ativo/ativo & $3,0(1,0 ; 6,0)$ & \multirow{3}{*}{$0,027^{d}$} \\
\hline Irregularmente ativo & $3,0(0,0 ; 5,0)$ & \\
\hline Sedentário & $3,0(1,0 ; 7,0)$ & \\
\hline \multicolumn{3}{|c|}{ Comorbidades associadas $^{\mathrm{a}}$} \\
\hline Nenhuma & $2,0(1,0 ; 4,0)$ & \multirow{3}{*}{$0,001^{\mathrm{d}}$} \\
\hline $1 \mathrm{a} 2$ & $2,0(1,0 ; 5,0)$ & \\
\hline Mais de 3 & $5,0(2,0 ; 7,0)$ & \\
\hline $\begin{array}{l}\text { a) Teste de Kruskal-Wallis: Autope } \\
\text { Nenhuma e } 1 \text { a 2 Nivivel de ativi } \\
\text { b) Teste' } U \text { 'de Mann-Whitney. } \\
\text { d) Diferença signnificativa: }<<0,0.5 \\
\text { Md = mediana. } \\
\text { UBS = unidade básica de saúde. }\end{array}$ & mentos - Mais de 2, com Nenhum e 1 & \\
\hline
\end{tabular}


Verificou-se (Tabela 3) correlação significativa $(\mathrm{p}<0,05)$ e inversa do indicativo de depressão com os dias de caminhada por semana $(\mathrm{r}=-0,18)$ e os minutos de caminhada por dia $(\mathrm{r}=-0,12)$. Tais acha- dos parecem indicar que a realização de atividades físicas leves está associada à redução dos indicativos de depressão.

\section{Tabela 3 - Correlação entre o nível de atividade física e o indicativo de depressão dos idosos usuários das unidades básicas de saúde, Maringá, Paraná, 2017}

\begin{tabular}{|c|c|c|c|c|c|c|c|c|c|c|}
\hline \multirow{2}{*}{ Variáveis } & \multicolumn{9}{|c|}{ Nível de atividade física } & \multirow{2}{*}{$\frac{\text { Depressão }}{10}$} \\
\hline & 1 & 2 & 3 & 4 & 5 & 6 & 7 & 8 & 9 & \\
\hline 1. & & $0,56^{\mathrm{a}}$ & $0,77^{a}$ & $0,23^{\mathrm{a}}$ & $0,14^{\mathrm{a}}$ & $0,18^{\mathrm{a}}$ & $-0,06$ & $-0,08$ & $-0,07$ & $-0,18^{a}$ \\
\hline 2. & & & $0,89^{\mathrm{a}}$ & $0,26^{a}$ & $0,31^{\mathrm{a}}$ & $0,28^{\mathrm{a}}$ & 0,02 & 0,01 & 0,01 & $-0,12^{\mathrm{a}}$ \\
\hline 3. & & & & $0,26^{\mathrm{a}}$ & $0,26^{\mathrm{a}}$ & $0,30^{\mathrm{a}}$ & 0,02 & 0,01 & 0,01 & 0,03 \\
\hline 4. & & & & & $0,86^{\mathrm{a}}$ & $0,93^{\mathrm{a}}$ & $0,47^{\mathrm{a}}$ & $0,47^{\mathrm{a}}$ & $0,47^{a}$ & $-0,02$ \\
\hline 5. & & & & & & $0,94^{\mathrm{a}}$ & $0,50^{\mathrm{a}}$ & $0,53^{\mathrm{a}}$ & $0,53^{\mathrm{a}}$ & 0,09 \\
\hline 6. & & & & & & & $0,51^{\mathrm{a}}$ & $0,53^{\mathrm{a}}$ & $0,53^{\mathrm{a}}$ & 0,05 \\
\hline 7. & & & & & & & & 0,98 & $0,98^{\mathrm{a}}$ & 0,06 \\
\hline 8. & & & & & & & & & $0,99^{\mathrm{a}}$ & 0,08 \\
\hline 9. & & & & & & & & & & 0,07 \\
\hline \multicolumn{11}{|l|}{10.} \\
\hline 11. & & & & & & & & & & \\
\hline
\end{tabular}

a) Correlação significativa: $p<0,05$.

Nota: 1. = dias de caminhada por semana; $2 .=$ minutos de caminhada $\mathrm{p} / \mathrm{dia} ; 3 .=$ minutos de caminhada $\mathrm{p} / \mathrm{semana} ; 4 .=$ dias de atividade moderada; $5 .=$ minutos de atividade moderada $\mathrm{p} /$ dia; 6 . minutos de atividade moderada $\mathrm{p} / \mathrm{semana} ; 7 .=$ dias de atividade vigorosa; $8 .=$ minutos de atividade vigorosa $\mathrm{p} / \mathrm{dia} ; 9 .=$ minutos de atividade vigorosa $\mathrm{p} / \mathrm{semana} ; 10 .=$ depressão.

\section{Discussão}

Os principais achados do presente estudo apontam que a renda mensal e as condições de saúde são fatores com o poder de intervir nos indicativos de depressão em idosos. Estes, quando praticavam atividade física regular, apresentavam menores indicativos de depressão quando comparados aos que a praticavam de forma irregular, e a maior duração da prática da caminhada estaria relacionada a baixos indicativos de depressão. Até onde foi possível verificar, este estudo traz resultado inédito no que diz respeito aos fatores intervenientes nos indicativos de depressão de idosos usuários das UBS de um munício do norte do Paraná.

Idosos praticantes de atividade física regular apresentaram menores indicativos de depressão. Idosos que realizam caminhada por mais tempo (em minutos) também apresentaram baixo indicativo de depressão. Estes dados são semelhantes aos encontrados por outro estudo, segundo o qual há forte associação entre o tempo gasto em atividades físicas e atividades de vida diária, e a depressão: $:^{12}$ idosos que passavam mais tempo da semana praticando atividade física e idosos mais ativos em atividades de vida diária, quando comparados a idosos que praticavam atividade física por menos tempo ou não a praticavam, apresentaram baixo indicativo de depressão. Barcelos-Ferreira et al., ${ }^{7}$ em estudo realizado com 1.563 idosos na cidade de São Paulo, evidenciaram que idosos fisicamente ativos mantêm um funcionamento saudável mais longo, quando comparados a seus pares sedentários, provavelmente resultado de melhora na função física e reforço do bem-estar psicológico.

0 histórico de quedas, a presença de três ou mais comorbidades e o uso de mais de dois medicamentos associou-se ao maior indicativo de depressão. A comorbidade representa um fator predisponente à ocorrência de quedas. ${ }^{13} 0$ risco de queda aumenta com 0 avançar da idade e sua ocorrência traz desfechos negativos ao idoso, entre os quais se destaca a depressão. Brito et al., ${ }^{14}$ em um estudo epidemio- 
lógico com 316 idosos brasileiros, observaram que indivíduos com sintomas depressivos apresentaram prevalência de queda $87 \%$ maior quando comparados aos assintomáticos para depressão.

Sob a perspectiva inversa, a depressão também pode aumentar a chance de queda quando se trata de efeito colateral dos medicamentos usados em tratamento, especialmente os fármacos como a serotonina e inibidores seletivos da receptação de benzodiazepina. ${ }^{14}$ No estudo de Moraes et al., ${ }^{15}$ a queda recorrente associa-se ao aumento significativo de comorbidades e depressão, sugerindo aumento do uso de medicamentos, diminuição de atividades físicas e atividades de vida diária, e tendência a diminuição da participação social, fatores concorrentes para depressão.

Pôde-se verificar, ademais, que a autopercepção de saúde ruim, somada ao uso de mais de dois medicamentos regulares, aumentou a proporção de sintomatologia depressiva entre os idosos estudados. Obviamente, o uso de medicamentos sugere, de fato, piora no quadro de saúde, justificando a autopercepção de saúde ruim; porém, quando associado ao indicativo de depressão, esse uso evidencia o quanto a saúde ruim impacta na qualidade de vida do idoso, com potencial para gerar sintomas psíquicos negativos.

A renda mensal até 3 salários mínimos correlacionou-se negativamente com o indicativo para depressão. Embora o presente estudo não tenha investigado o arranjo familiar dos indivíduos participantes, a literatura tem evidenciado, especificamente nas famílias de baixa renda, a importância central da contribuição do idoso na economia familiar. Segundo o estudo realizado por Rabelo e Neri, ${ }^{13}$ entre os 134 idosos estudados, $41 \%$ viviam com descendentes, $23 \%$ com o cônjuge e descendentes, $17,9 \%$ sozinhos, $9,7 \%$ em outros tipos de arranjo familiar e 7,5\% somente com o cônjuge. De todos os idosos analisados, $72,4 \%$ exerciam o papel de chefe de família, contribuindo total $(49,2 \%)$ ou parcialmente (44\%) para o sustento de seu núcleo familiar. ${ }^{16}$

Considerando-se que a principal, senão a única fonte de renda do idoso brasileiro, é a aposentadoria ou a pensão por morte do cônjuge, o valor do benefício da Previdência Pública torna-se a única ou principal fonte de renda das famílias. ${ }^{17} \mathrm{~A}$ restrição de recursos financeiros deixa 0 idoso em uma situação de vulnerabilidade social, risco de adoecimento, agravamento de enfermidades e agravos preexistentes e dificuldade de manejo de sua condição de saúde, devido à escassez de recursos para aquisição de medicamentos não fornecidos por farmácia popular. ${ }^{17}$ 0 contexto de pobreza também pode gerar estresse crônico e afetar os domínios físico e cognitivo do indivíduo, particularmente do idoso.

Não obstante os importantes resultados, o estudo em tela apresenta limitações. Deve-se ter cuidado ao extrapolar os achados para a população geral, visto que a amostra é composta por indivíduos residentes em áreas de abrangência de UBS de um único município, e não implica generalizações para a população brasileira. Por fim, o fato de tratar-se de um estudo transversal impede a avaliação de relações diretas de causalidade entre as variáveis estudadas. Sugere-se a realização de pesquisas semelhantes em outros municípios brasileiros com idosos usuários de UBS, como também com idosos não usuários de UBS, para comparações dos resultados.

Os achados deste trabalho permitem concluir que a renda mensal e as condições de saúde são fatores intervenientes nos indicativos de depressão em idosos. Ressalta-se que os idosos com maior poder aquisitivo e melhores condições de saúde possuem menor tendência a apresentar depressão, e que a prática de atividades físicas leves, como a caminhada, está associada à baixa tendência de depressão na terceira idade.

\section{Contribuição dos autores}

Oliveira DV contribuiu na concepção e delineamento do estudo, coleta de dados, escrita científica e revisão crítica do conteúdo do manuscrito. Pivetta NRS contribuiu com a escrita científica. Oliveira GVN e Silva DA participaram da coleta de dados, escrita científica e revisão crítica do conteúdo do manuscrito. Nascimento Júnior JRA participou da análise de dados e correção da versão final do manuscrito. Cavaglieri CR contribuiu com a orientação geral da pesquisa e correção final do manuscrito. Todos os autores aprovaram a versão final do manuscrito e são responsáveis por todos os seus aspectos, incluindo a garantia de sua precisão e integridade. 


\section{Referências}

1. Neves RT, Laham CF, Aranha VC, Santiago A, Ferrari S, Lucia MCS. Envelhecimento e doenças cardiovasculares: depressão e qualidade de vida em idosos atendidos em domicílio. Psicol Hosp [Internet]. 2013 jul [citado 2019 jun 5];11(2):7298. Disponível em: http://pepsic.bvsalud.org/pdf/ph/ v11n2/v11n2a06.pdf

2. Ferreira OGL, Maciel SC, Costa SMG, Silva AO, Moreira M. Envelhecimento ativo e sua relação com a independência funcional. Texto Contexto Enferm [Internet]. 2012 jul-set [citado 2019 jun 5];21(3):513-8. Disponível em: http://www.scielo.br/ $\mathrm{pdf} / \mathrm{tce} / \mathrm{v} 21 \mathrm{n} 3 / \mathrm{v} 21 \mathrm{n} 3 \mathrm{a} 04$.pdf. doi: 10.1590/S010407072012000300004

3. United Nations. Department of Economic and Social Affairs Population Division. World population ageing 2015 [Internet]. New York: United Nations; 2015 [cited 2019 Jun 5]. 149 p. Available from: https:// www.un.org/en/development/desa/population/ publications/pdf/ageing/WPA2015_Report.pdf

4. Lima AMP, Ramos JLS, Bezerra IMP, Rocha RPB, Batista HMT, Pinheiro WR. Depressão em idosos: uma revisão sistemática da literatura. Rev Epidemiol Controle Infecç [Internet]. 2016 abr-jun [citado 2019 jun 5];6(2):96-103. Disponível em: https:// online.unisc.br/seer/index.php/epidemiologia/article/ view/6427. doi: 10.17058/reci.v6i2.6427

5. Cunha RVD, Bastos GAN, Del Duca GF. Prevalência de depressão e fatores associados em comunidade de baixa renda de Porto Alegre, Rio Grande do Sul. Rev Bras Epidemiol [Internet]. 2012 jun [citado 2019 jun 5];15(1):346-54. Disponível em: http://www. scielo.br/scielo.php?script $=$ sci_arttext\&pid $=\mathrm{S} 1415-$ 790X2012000200012. doi: 10.1590/S1415790X2012000200012

6. Governo Federal (BR). Programa mais médicos [Internet]. Brasília: Governo Federal; 2017 [citado 2017 dez 17]. Disponível em: http://maismedicos.gov. br/o-que-tem-na-ubs

7. Barcelos-Ferreira R, Nakano EY, Steffens DC, Bottino CM. Quality of life and physical activity associated to lower prevalence of depression in communitydwelling elderly subjects from Sao Paulo. J Affect Disord [Internet]. 2013 Sep [cited 2019 Jun 5];150(2):616-22. Available from: https://www.ncbi. nlm.nih.gov/pubmed/23499164. doi: 10.1016/j. jad.2013.02.024
8. Folstein M, Folstein S, Mchugh P. Mini exame do estado mental: um método prático para classificar 0 estado cognitivo dos pacientes para o clínico. J Pesqui Psiquiátrica. 1975;12(1):1-10.

9. Brucki SM, Nitrini R, Caramelli P, Bertolucci PHF, Okamoto IH. Sugestões para o uso do mini-exame do estado mental no Brasil. Arq Neuro-Psiquiatr [Internet]. 2003 set [citado 2019 jun 5];61(3):777-81. Disponível em: http:// www.scielo.br/pdf/anp/v61n3B/17294.pdf. doi: 10.1590/S0004-282X2003000500014

10. Matsudo S, Araújo T, Matsudo V, Andrade D, Andrade E, Oliveira LC, et al. Questionário internacional de atividade física (Ipaq): estupo de validade e reprodutibilidade no Brasil. Rev Bras Ativ Fís Saúde [Internet]. 2001 [citado 2019 jun 5];6(2):5-18. Disponível em: http://rbafs. org.br/RBAFS/article/view/931. doi: 10.12820/ rbafs.v.6n2p5-18

11. Yesavage JA, Brink TL, Rose TL, Lum 0, Huang V, Adey M, et al. Development and validation of a geriatric depression screening scale: a preliminary report. J Psychiat Res. 1983;17(1):37-49.

12. Bhamani MA, Khan MM, Karim MS, Mir MU. Depression and its association with functional status and physical activity in the elderly in Karachi, Pakistan. Asian J Psychiatr [Internet]. 2015 Apr [cited 2019 Jun 5];14:46-51. Available from: https://www.sciencedirect.com/science/article/pii/ S1876201814002834?via\%3Dihub. doi: 10.1016/j. ajp.2014.12.004

13. Guimarães LH, Galdino DC, Martins FL, Vitorino DF, Pereira KL, Carvalho EM. Comparação da propensão de quedas entre idosos que praticam atividade física e idosos sedentários. Rev Neurocienc [Internet]. 2004 abr-jun [citado 2019 jun 5];12(2):68-72. Disponível em: http:// revistaneurociencias.com.br/edicoes/2004/RN\%20 12\%2002/Pages\%20from\%20RN\%2012\%2002-2. pdf

14. Brito TA, Coqueiro RS, Fernandes MH, Jesus CS. Determinants of alls in Community Dwelling elderly: Hierarchical analysis. Public Health Nurs [Internet]. 2014 Jul-Aug [cited 2019 Jun 5];31(4):290-7. Available from: https:// onlinelibrary.wiley.com/doi/abs/10.1111/ phn.12126. doi: 10.1111/phn.12126 
15. Moraes SA, Soares WJS, Lustosa LP, Bilton TL, Ferrioli E, Perracini MR. Características das quedas em idosos que vivem na comunidade: estudo de base populacional. Rev Bras Geriatr Gerontol [Internet]. 2017 set-out [citado 2019 jun 5];20(5):693-704. Disponível em: http://www. redalyc. org/articulo.oa? $\mathrm{id}=403853542011$. doi: 10.1590/1981-22562017020.170080

16. Rabelo DF, Neri AL. Family configuration and physical and psychological health status in a sample of elderly. Cad Saúde Pública [Internet]. 2015 Apr [cited 2019 Jun 5];31(4):874-84.

\begin{abstract}
Objective: to analyze factors influencing depression markers in elderly patients at primary bealthcare centers (PHC) in the city of Maringá, Paraná, Brazil, in 2017. Methods: this was a cross-sectional study carried out with elderly individuals at PHCs in Maringá city; we used a questionnaire comprising sociodemographic questions, the Geriatric Depression Scale (GDS) and the International Physical Activity Questionnaire (IPAQ); we analyzed associations and compared depression markers with sociodemographic variables and health conditions. Results: 645 elderly people took part in the study; those with the highest depression markers had lower income, poor health perception, a bistory of falls and three or more comorbidities; the physically active elderly had lower depression markers. Conclusion: monthly income and health conditions are factors that influence depression markers; doing light physical activities is associated with lower tendency of depression in the elderly.
\end{abstract}

Keywords: Motor Activity; Depression; CrossSectional Studies; Geriatrics; Aging.
Available from: http://www.scielo.br/pdf/csp/ v31n4/0102-311X-csp-31-04-00874.pdf. doi: 10.1590/0102-311X00087514

17. Santos-Orlandi AA, Brito TRP, Ottaviani AC, Rossetti ES, Zazzetta MS, Gratão ACM, et al. Perfil de idosos que cuidam de outros idosos em contexto de alta vulnerabilidade social. Escola Anna Nery [Internet]. 2017 [citado 2019 jun 5];21(1):e20170013. Disponível em: http:// www.scielo.br/pdf/ean/v21n1/en_1414-8145ean-21-01-e20170013.pdf. doi: 10.5935/14148145.20170013

\section{Resumen}

objetivo: analizar los factores intervinientes en los indicativos de depresión en adultos mayores de las unidades básicas de salud (UBS) del municipio de Maringá, Paraná, Brasil, en 2017. Métodos: estudio transversal realizado con adultos mayores usuarios de las UBS del municipio de Maringá; se utilizó un cuestionario con preguntas sociodemográficas, la Escala de Depresión Geriátrica (Geriatric Depression Scale [GDS]) y el Cuestionario Internacional de Actividad Física (International Physical Activity Questionnaire [IPAQ]); se analizaron las asociaciones y comparaciones del indicativo de depresión con las variables sociodemográficas y las condiciones de salud. Resultados: los adultos mayores que presentaron mayor indicativo de depresión fueron los con menores ingresos, percepción de mala salud, histórico de caídas y tres o más comorbilidades; los adultos mayores activos físicamente presentaron menor indicativo de depresión. Conclusión: la renta mensual y las condiciones de salud son factores intervinientes en los indicativos de depresión; la práctica de actividades físicas leves está asociada a menor tendencia de depresión en la tercera edad.

Palabras clave: Actividad Motora; Depresión; Estudios Transversales; Geriatrí; Envejecimiento.

Recebido em 15/05/2018

Aprovado em 06/05/2019

Editora associada:Suele Manjourany Duro - — orcid.org/0000-0001-5730-0811 